\title{
CENTERS OF MASS FOR OPERATOR-FAMILIES
}

\author{
by MAKOTO TAKAGUCHI
}

(Received 5 November, 1990)

1. Introduction. Let $H$ be a complex Hilbert space and let $B(H)$ be the algebra of (bounded) operators on $H$. Let $A=\left(A_{1}, \ldots, A_{n}\right)$ be an $n$-tuple of operators on $H$. The joint numerical range of $A$ is the subset $W(A)$ of $\mathbb{C}^{n}$ such that

$$
W(A)=\left\{\left(\left(A_{1} x, x\right), \ldots,\left(A_{n} x, x\right)\right): x \in H,\|x\|=1\right\} .
$$

We now describe several definitions of joint spectra of a commuting $n$-tuple $A$ of operators (see [3]): the approximate point spectrum

$$
\begin{array}{r}
\sigma_{\pi}(A)=\left\{\lambda \in \mathbb{C}^{n}: \text { there exists a sequence }\left\{x_{i}\right\} \text { of unit vectors in } H\right. \\
\text { such that } \left.\left\|\left(A_{k}-\lambda_{k}\right) x_{i}\right\| \rightarrow 0 \text { as } i \rightarrow \infty, k=1, \ldots, n\right\},
\end{array}
$$

the left spectrum

$$
\sigma_{l}(A)=\left\{\lambda \in \mathbb{C}^{n}: A-\lambda \text { generates a proper left ideal in } B(H)\right\},
$$

the right spectrum

$$
\sigma_{r}(A)=\left\{\lambda \in \mathbb{C}^{n}: A-\lambda \text { generates a proper right ideal in } B(H)\right\},
$$

the Harte spectrum

$$
\sigma_{H}(A)=\sigma_{l}(A) \cup \sigma_{r}(A),
$$

the commutant spectrum

$$
\sigma^{\prime}(A)=\left\{\lambda \in \mathbb{C}^{n}: A-\lambda \text { generates a proper ideal in } A^{\prime}\right\},
$$

the double commutant spectrum

$$
\sigma^{\prime \prime}(A)=\left\{\lambda \in \mathbb{C}^{n}: A-\lambda \text { generates a proper ideal in } A^{\prime \prime}\right\}
$$

(where $A^{\prime}$ and $A^{\prime \prime}$ are the commutant and double commutant of $A$ in $B(H)$, respectively), the Taylor spectrum

$$
\begin{gathered}
\sigma_{T}(A)=\left\{\lambda \in \mathbb{C}^{n}: \text { the Koszul complex } E(A-\lambda, H) \text { on } H\right. \text { associated } \\
\text { with } A-\lambda \text { is not exact }\}
\end{gathered}
$$

(see [7] or [8]) and the polynomial spectrum

$$
\sigma_{P}(A)=\left\{\lambda \in \mathbb{C}^{n}: p(\lambda) \in \sigma(p(A)) \text { for all } n \text {-variate polynomials } p\right\}
$$

(of course, $\sigma(p(A))$ is the usual spectrum of $p(A) \in B(H)$ ). Note that $\sigma_{\pi}, \sigma_{l}, \sigma_{r}$ and $\sigma_{H}$ can be defined even if $A$ is not commuting.

The joint operator norm, joint numerical radius and joint spectral radii of $A$, denoted by $\|A\|, w(A)$ and $r .(A)$ respectively, are defined by

$$
\begin{gathered}
\|A\|=\sup \left\{\left(\sum_{k=1}^{n}\left\|A_{k} x\right\|^{2}\right)^{1 / 2}:\|x\|=1\right\}, \\
w(A)=\sup \left\{\left(\sum_{k=1}^{n}\left|\left(A_{k} x, x\right)\right|^{2}\right)^{1 / 2}:\|x\|=1\right\}
\end{gathered}
$$

Glasgow Math. J. 34 (1992) 123-126. 
and

$$
r .(A)=\sup \{|\lambda|: \lambda \in \sigma .(A)\}
$$

respectively, where $\sigma .=\sigma_{\pi}, \sigma_{l}, \sigma_{H}, \sigma^{\prime}, \sigma^{\prime \prime}, \sigma_{T}$ or $\sigma_{P}$ and $r .=r_{\pi}, r_{l}, r_{H}, r^{\prime}, r^{\prime \prime}, r_{T}$ or $r_{P}$. Note that we define the joint operator norm, joint numerical radius, $r_{\pi}, r_{l}$ and $r_{H}$ for all (not necessarily commuting) $n$-tuples of operators, but we define $r^{\prime}, r^{\prime \prime}, r_{T}$ and $r_{P}$ only for commuting $n$-tuples of operators.

We shall call $A$ jointly normaloid if $\|A\|=w(A)$, and call $A$ jointly transloid if $A-z$ is jointly normaloid for any point $z \in \mathbb{C}^{n}$.

In this note we shall define the center of mass for an $n$-tuple of operators and state that the center of mass of $A$ is coincident with the center of the smallest sphere containing the joint spectrum of $A$ in case of a jointly transloid $n$-tuple $A=\left(A_{1}, \ldots, A_{n}\right)$ of operators. The center of mass of a single operator has been defined by Stampfli [5].

\section{Results.}

THEOREM 1. For an $n$-tuple $A=\left(A_{1}, \ldots, A_{n}\right)$ of operators the following conditions are equivalent:

(i) $\|A\|^{2}+|\lambda|^{2} \leqq\|A-\lambda\|^{2}$ for all $\lambda \in \mathbb{C}^{n}$;

(ii) $\|A\| \leqq\|A+\lambda\|$ for all $\lambda \in \mathbb{C}^{n}$.

Proof. It is clear that (i) implies (ii). So we shall show that (ii) implies (i). For any natural number $m$, it follows that

$$
\begin{aligned}
\|A-\lambda\|^{2}-\|A\|^{2}= & \sup \left\{\sum_{k=1}^{n}\left\|\left(A_{k}-\lambda_{k}\right) x\right\|^{2}:\|x\|=1\right\}+(m-1) \sup \left\{\sum_{k=1}^{n}\left\|A_{k} x\right\|^{2}:\|x\|=1\right\} \\
& -m \sup \left\{\sum_{k=1}^{n}\left\|A_{k} x\right\|^{2}:\|x\|=1\right\} \\
\geqq & \sup \left\{\sum_{k=1}^{n}\left(\left(\left(A_{k}-\lambda_{k}\right)^{*}\left(A_{k}-\lambda_{k}\right)+(m-1) A_{k}^{*} A_{k}\right) x, x\right):\|x\|=1\right\} \\
-m \sup \left\{\sum_{k=1}^{n}\left\|\left(A_{k}-\frac{\lambda_{k}}{m}\right) x\right\|^{2}:\|x\|=1\right\}=\sup \left\{\sum _ { k = 1 } ^ { n } \left(\left(m\left(A_{k}-\frac{\lambda_{k}}{m}\right)^{*}\left(A_{k}-\frac{\lambda_{k}}{m}\right)\right.\right.\right. & \\
& \left.\left.\left.+\frac{m-1}{m}\left|\lambda_{k}\right|^{2}\right) x, x\right):\|x\|=1\right\}-m \sup \left\{\sum_{k=1}^{n}\left\|\left(A_{k}-\frac{\lambda_{k}}{m}\right) x\right\|^{2}:\|x\|=1\right\} \\
= & \sup \left\{\sum_{k=1}^{n} m\left(\left(A_{k}-\frac{\lambda_{k}}{m}\right)^{*}\left(A_{k}-\frac{\lambda_{k}}{m}\right) x, x\right):\|x\|=1\right\} \\
& +\frac{m-1}{m} \sum\left|\lambda_{k}\right|^{2}-m \sup \left\{\sum\left\|\left(A_{k}-\frac{\lambda_{k}}{m}\right) x\right\|^{2}:\|x\|=1\right\} \\
= & \frac{m-1}{m} \sum\left|\lambda_{k}\right|^{2}=\frac{m-1}{m}|\lambda|^{2} .
\end{aligned}
$$

Thus the proof is complete.

THeorem 2. Given $A=\left(A_{1}, \ldots, A_{n}\right)$, there exists a unique $z_{0} \in \mathbb{C}^{n}$, such that

$$
\left\|A-z_{0}\right\| \leqq\|A-\lambda\| \text { for all } \lambda \in \mathbb{C}^{n} \text {. }
$$


Proof. Since $\|A-\lambda\|$ is large for $\lambda$ large, $\inf \left\{\|A-\lambda\|: \lambda \in \mathbb{C}^{n}\right\}$ must be attained at some point, say $z_{0}$. The uniqueness of $z_{0}$ is deduced from the above theorem.

Definition 3. For $A=\left(A_{1}, \ldots, A_{n}\right)$, we define the center of mass of $A$ to be the point $z_{0}$ specified in Theorem 2 , and designate it by $c_{A}$.

TheOREM 4. Let $A=\left(A_{1}, \ldots, A_{n}\right)$ be commuting and jointly transloid, and let $\sigma .(A)$ be $\sigma_{\pi}(A), \sigma_{l}(A), \sigma_{H}(A), \sigma_{T}(A), \sigma^{\prime}(A), \sigma^{\prime \prime}(A)$ or $\sigma_{P}(A)$. Then $c_{A}$ is the center of the smallest closed ball containing $\sigma .(A)$.

Proof. It is well known that $\sigma_{\pi}(A)=\sigma_{l}(A) \subset \sigma_{H}(A) \subset \sigma_{T}(A) \subset \sigma^{\prime}(A) \subset \sigma^{\prime \prime}(A) \subset$ $\sigma_{P}(A)$ (see [8], [7], [1]). Moreover, from Theorem 2.5.4 of [4] and Corollary 2 of Proposition 1.1 .2 of $[\mathbf{1}]$, it follows that $\sigma_{P}(A) \subset$ co $\overline{W(A)}$, the convex hull of the closure of $W(A)$. Consequently, $r_{\pi}(A)=r_{l}(A) \leqq r_{H}(A) \leqq r_{T}(A) \leqq r^{\prime}(A) \leqq r^{\prime \prime}(A) \leqq r_{P}(A) \leqq w(A) \leqq$ $\|A\|$. On the other hand, if $A$ is jointly normaloid, then $\|A\|=r_{\pi}(A)$ (see [6]). Consequently, if $A$ is jointly transloid, then $r_{\pi}(A-z)=r_{l}(A-z)=r_{H}(A-z)=$ $r_{T}(A-z)=r^{\prime}(A-z)=r^{\prime \prime}(A-z)=r_{P}(A-z)=\|A-z\|$ for every $z \in \mathbb{C}^{n}$. Thus we have $\sup \left\{\left|z-c_{A}\right|: z \in \sigma .(A)\right\}=\left\|A-c_{A}\right\| \leqq\|A-\lambda\|=\sup \{|z-\lambda|: z \in \sigma .(A)\}$ for each $\lambda \in$ $\mathbb{C}^{n}$. Thus the proof is complete.

Remarks. (i) In Theorem 4 , the condition that $A$ is commuting is not necessary in respect of $\sigma_{\pi}(A), \sigma_{l}(A)$ and $\sigma_{H}(A)$.

(ii) The class of jointly transloid operator-families includes the following kinds of classes of operator-families (see [2], [6]).

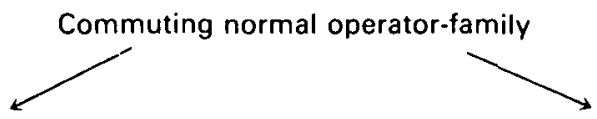

Doubly commuting quasinormal operator-family

Family of commuting operators such that the polynomial spectrum of it is a complete spectral set in Arveson's sense<smiles>C1CC1</smiles>

Doubly commuting subnormal operator-family

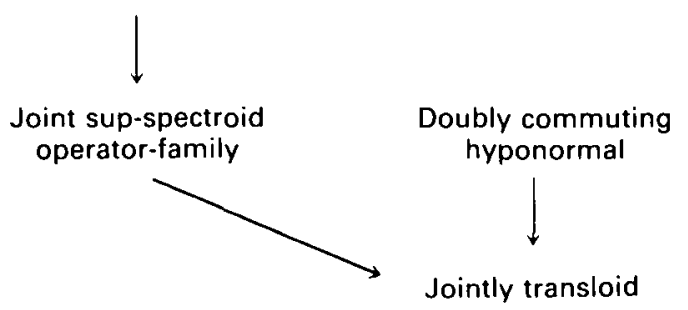

Family of commuting operators such that the convex hull of the closure of the joint numerical range of it is a complete spectral set

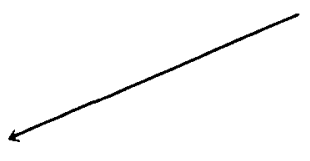

ACKNOWLEDGEMENT. I would like to express my gratitude to Professor Yoshihiro Nakamura for his help.

\section{REFERENCES}

1. W. Arveson, Subalgebras of $C^{*}$-algebras II, Acta Math. 128 (1972), 271-308.

2. M. Chō and M. Takaguchi, Some classes of commuting $n$-tuples of operators, Studia Math. 80 (1984), 245-259. 
3. R. E. Curto, Connections between Harte and Taylor spectra, Rev. Roumaine Math. Pures Appl. 31 (1986), 203-215.

4. N. P. Dekker, Joint numerical range and joint spectrum of Hilbert space operators (Ph.D. thesis, University of Amsterdam, 1969).

5. J. G. Stampfli, the norm of a derivation, Pacific J. Math. 33 (1970), 737-747.

6. M. Takaguchi and M. Chō, Joint normaloid operator-family, Sci. Rep. Hirosaki Univ. 27 (1980), 50-59.

7. J. L. Taylor, A joint spectrum for several commuting operators, J. Funct. Anal. 6 (1970), 172-191.

8. F.-H. Vasilescu, A characterization of the joint spectrum in Hilbert spaces, Rev. Roumaine Math. Pures Appl. 22 (1977), 1003-1009.

Department of Mathematics

Faculty of Science

HiRosaki UNIVERSITY

HiRosaki

036 JAPAN 